\title{
General anesthesia for an adolescent with Pelizaeus-Merzbacher disease - A case report -
}

Received May 4, 2018

Revised 1st, June 15, 2018 2nd, June 18, 2018 Accepted June 22, 2018

\section{Corresponding author}

Hyuckgoo Kim, M.D.

Department of Anesthesiology and Pain Medicine, Yeungnam University College of Medicine, 170 Hyeonchung-ro, Nam-gu, Daegu 42415, Korea

Tel: 82-53-620-3357

Fax: 82-53-626-5275

E-mail: rlagurrn@hanmail.net ORCID

https://orcid.org/0000-0002-3879-6441

\section{Hyuckgoo Kim and Chaeseok Lim}

Department of Anesthesiology and Pain Medicine, Yeungnam University College of Medicine, Daegu, Korea

Pelizaeus-Merzbacher disease (PMD) is a progressive and degenerative chromosomal disorder of the central nervous system caused by defective myelin production. Few case reports have been issued on the anesthetic management of PMD, because of its extremely low incidence. We anesthetized a 13-year-old female patient diagnosed with PMD for ophthalmic surgery because of intermittent exotropia. General anesthesia was induced and maintained with propofol and sevoflurane in air and oxygen. Rocuronium was administered to facilitate orotracheal intubation, and residual neuromuscular blockage was reversed with pyridostigmine. Between emergence to 24 hours postoperatively, her muscle power completely recovered and no unpredictable events occurred. Summarizing, anesthesiologists should be concerned about the high possibility of aspiration, spasticity, and seizure during the perioperative period in patients with even mild PMD. Appropriate preoperative evaluation, intraoperative monitoring, and choice of proper anesthetic drugs enable safe anesthesia in patients with PMD.

Keywords: General anesthesia; Genetic disease; Leukodystropy; Pelizaeus-Merzbacher disease.
Pelizaeus-Merzbacher disease (PMD) is a progressive, degenerative chromosomal disorder of the central nervous system (CNS) caused by defective myelin production. PMD is a variant of leukodystrophy, which is characterized by neuromuscular dysfunctions of the CNS. Thus, the clinical symptoms of PMD arise from widespread hypomyelination of the nervous system and include developmental motor disability, ataxia, choreoathetosis, and cognitive impairment. In addition, an anesthesiologist must consider the possibility of abnormal muscle tone, such as spasticity, airway complications due to pharyngeal muscle weakness, and the risks of aspiration and seizure.

Only a few case reports have been published on the anesthetic management of patients with PMD because of its low prevalence [1], which is about 0.13 or 1.45 per 100,000 live births [2,3]. Here we present the case of a 13-year-old girl with PMD who underwent elective ophthalmic surgery under general anesthesia.

\section{CASE REPORT}

A 13-year-old girl required general anesthesia for resection and recession of ocular muscles because of intermittent exotropia. Her mental development was delayed by about five years, and she exhibited snoring while sleeping and mild ataxia. She had been diagnosed with PMD by genetic testing at eight years and had been followed twice per annum at a descriptor hospital. However, she had not been medicated for PMD and experienced hypotonia, spasticity, dystonic posturing, and seizures. She had no history of epilepsy, aspi-

This is an Open Access article distributed under the terms of the Creative Commons Attribution Non-Commercial License (http://creativecommons.org/licenses/by-nc/4.0) which permits unrestricted non-commercial use, distribution, and reproduction in any medium, provided the original work is properly cited. 
ration pneumonia, or surgery.

On preoperative examination, she was $149 \mathrm{~cm}$ high, weighed $47 \mathrm{~kg}$, and had a blood pressure of 130/90 $\mathrm{mmHg}$, a heart rate of 100 beats/min, percutaneous oxygen saturation of $100 \%$, and a body temperature of $36.7^{\circ} \mathrm{C}$. Preoperative blood test, urinalysis, chest radiography, and electrocardiography findings were unremarkable. She revealed no respiratory distress, such as nose flaring, grunting, wheezing, or ncreased breathing rate. A peripheral intravenous line was placed on an extremity in a ward but premedication was not administered. She exhibited a neutral facial expression in the preoperative holding area, but appeared sad with teary eyes due to separation from her mother when transferred to the operating room.

On her arrival in the operating room, her blood pressure was 150/90 $\mathrm{mmHg}$ and heart rate was 95 beats/min. Standard anesthetic monitoring, including electrocardiography, pulse oximetry, noninvasive blood pressure, and end-tidal $\mathrm{CO}_{2}$, was initiated. In addition, bispectral index (BIS), esophageal thermometry, and train-of-four (TOF) monitoring were instituted. General anesthesia was induced with $100 \mathrm{mg}$ of propofol and sevoflurane in air and oxygen. About one minute after administration of $30 \mathrm{mg}$ of rocuronium, TOF decreased to zero. Tracheal intubation was performed without airway trauma or hemodynamic instability. General anesthesia was maintained with sevoflurane (0.7 MAC) in air and oxygen with continuous intravenous remifentanil $(0.05 \mu \mathrm{g} / \mathrm{kg} / \mathrm{min})$. BIS was maintained at $\sim 55$ during the operation. TOF ratio reached 2 at around 20 minutes after administration of rocuronium and recovered by more than $70 \%$ approximately 40 minutes later. Body temperature was around $36.8^{\circ} \mathrm{C}$ during general anesthesia. Before extubation, residual neuromuscular blockage was reversed with pyridostigmine $10 \mathrm{mg}$ and glycopyrrolate $0.4 \mathrm{mg}$. During emergence from anesthesia, TOF ratio returned to $100 \%$, and no unpredictable events were encountered.

Anesthesia lasted 70 minutes and was completed uneventfully. No significant hemodynamic or respiratory system changes occurred. The patient was transferred to a postanesthesia care unit and remained there for 30 minutes. She exhibited no signs of respiratory depression, and her muscle power almost completely recovered. During the 24 hours following surgery, no adverse events occurred, and she was discharged at two days postoperation.

\section{DISCUSSION}

Patients with developmental delays caused by failure of white-matter myelination are diagnosed with leukodystrophy, a progressive, degenerative disease of cerebral white matter caused by defective myelin production; it thus differs from demyelinating diseases, like multiple sclerosis. The leukodystrophic diseases include metachromatic leukodystrophy, adrenoleukodystrophy, Krabbe's disease, Canavan's disease, Alexander's disease, and PMD [4]. PMD is caused by abnormalities in the proteolipid protein 1 gene (PLP1) and exhibits an X-linked recessive pattern, as identified by genomic analysis [5]. PLP1 is the dominant protein in CNS myelin and constitutes $50 \%$ of myelin-sheath protein. Mutation of PLP1 may lead to the complete absence of myelin sheaths or profound loss of oligodendrocytes, which are the myelinforming cells in the CNS [6]. Myelin sheaths are essential for the conduction of electric signals in the nervous system, because they facilitate rapid conduction of action potentials along nerve fibers.

Our patient was a 13-year-old female with PMD, but without any other underlying disease, who underwent ophthalmic surgery under general anesthesia. She had been diagnosed with PMD at eight years old and had not experienced hypotonia, spasticity, dystonic posturing, or seizures. In addition, she had no history of aspiration pneumonia or gastroesophageal reflux disease, and had no feeding difficulty, stridor, or nystagmus. Despite the absence of such symptoms, in a patient with PMD, the response to general anesthesia is unpredictable, especially when the disease interferes with neuromuscular function. In addition, lack of experience in using general anesthesia for PMD patients makes the anesthesiologist monitor the patients more carefully. For these reasons, we considered that the perioperative risks of anesthetic complications might be similar to those of PMD in its usual course.

In usual PMD, the anesthesiologist should concentrate on preventing anesthetic complications related to abnormal muscle tone, such as spasticity, airway complications due to pharyngeal muscle weakness, risk of aspiration, and seizure. Especially, if there is more risk of aspiration because of gastroesophageal reflux and pharyngeal hypotonia, rapid sequence induction and emergence are essential. In addition, antacids, antiemetic drugs, and gastric stimulants may be 
considered [7]. During the emergence period, exacerbation of spasticity is the most common complication associated with PMD and may remain after the neuromuscular blockage has subsided. In some cases, spasticity has led to muscle contractures, which in turn cause respiratory insufficiency, hyperpyrexia, dehydration, and rhabdomyolysis [8]. If exacerbation of spasticity is noted, the administration of benzodiazepine may provide effective management [9]. Seizures also increase the risk with general anesthesia. Avoiding a high concentration of anesthetics and hyperventilation may prevent seizures.

It has been suggested that all anesthetic drugs can be used for patients with PMD [8]. In our patient, we used propofol, sevoflurane, and remifentanil for the induction and maintenance of anesthesia. Propofol was chosen for induction because of its strong anticonvulsant effects and sevoflurane for maintenance because it provides rapid emergence and recovery, as is appropriate for short surgery. BIS monitoring is useful to maintain anesthesia depth and helps prevent excessive anesthetic administration. However, it was recently reported that the reliability of BIS monitoring decreases in severe cases of PMD because of possible EEG abnormalities [10]. In PMD, neuromuscular blocking-agent selection is controversial, and we considered a depolarizing neuromuscular blocking agent because rapid sequence induction and rapid emergence are essential, given the risk of aspiration. However, such an agent can cause unpredictable responses, and massive hyperkalemia developed after the administration of succinylcholine in a patient with upper motor neuron disease [11]. We chose rocuronium as the neuromuscular block. The patient exhibited normal neuromuscular function as confirmed by TOF monitoring from induction to the postanesthetic care unit. Furthermore, drug onset time and time to recovery from the nondepolarizing neuromuscular blocking agent, rocuronium, were similar to those of a healthy adolescent. TOF monitoring is essential for confirming neuromuscular function recovery and the detection of spasticity.

During general anesthesia, our patient did not exhibit any sign or symptom of adverse effects associated with general anesthesia, such as, respiratory depression or spasticity. PMD has various clinical courses according to genomic mutation types. We were not able to identify the genomic mutation type of PMD in our patient, because her family did not want to inform us of additional medical history. However, we believe it to have been a mild form of PMD, given the observed symptoms. In addition, PMD in female patients is a very rare possibility, because the disorder exhibits an X-linked recessive pattern.

PMD can be classified based on clinical findings or genomic mutation type. According to a classification devised by Renier et al. [12], PMD may be classified as connatal, transitional, classical, or X-linked severe spastic paraplegia (SPG) types. Connatal PMD exhibits more severe clinical features than do the other types; such patients develop symptoms during infancy and die during infancy or childhood. Clinical manifestation in patients with this type of PMD include feeding problems, a whistling breathing sound, progressive spasticity, dysarthria, ataxia, and seizures. In contrast, classic PMD is a milder form, with an onset in adolescence, which is followed by progressive deterioration into adulthood. Classic PMD and SPG are allelic disorders and share clinical features, including nystagmus, cerebellar ataxia, and pyramidal syndrome [13], though myelination is not severely disrupted in the SPG type, and symptomatically the disease may be limited to lower-limb spasticity. The transitional form is similar to the connatal type but its course is not rapid.

A PLP1 mutation type has been shown to be related to clinical severity and to affect axo-glial morphology and neuronal physiology, which are involved in the pathogenesis of PMD [14]. Excessive PLP1 production, which is caused by PLP1 duplications, disturbs its transport and results in its accumulations in endosomes and lysosomes and in the initiation of apoptosis. Two-thirds of PMD patients have been reported to harbor PLP1 duplication and present typical features of classic PMD [15]. The proteins containing missense mutations become trapped in the endoplasmic reticulum (ER), which causes ER stress and oligodendrocyte death. Connatal type PMD is associated with missense mutations. PLP1 has also been reported to display a partial duplication mutation, a point mutation, and insertion and deletion mutations. A patient with the partial PLP1 duplication showed null expression and manifested a milder form of PMD. Loss of PLP1 does not cause oligodendrocyte cell death, but rather forms immature myelin [15]. Therefore, deletions of the entire PLP1 gene can lead to the rare, mild PMD and SPG types. The female adolescent probably had each mutated gene related to PLP1 on the X-chromosome. In addition, the genomic mutation types may be partial duplication or null genomic mutations, resulting in PLP1 loss. 
In conclusion, we have described the anesthetic management of a 13-year-old female with PMD. Patients with PMD may exhibit a broad range of clinical severity. In this case, the patient had a mild form of PMD. For general anesthesia, anesthesiologists should consider the high prevalence of seizure, spasticity, and aspiration in PMD patients. Appropriate evaluations of symptoms and signs before anesthesia, intraoperative monitoring, and proper choice of anesthetic drugs enable the safe provision of anesthesia to patients with PMD.

\section{CONFLICTS OF INTEREST}

No potential conflict of interest relevant to this article was reported.

\section{ORCID}

Chaeseok Lim: https://orcid.org/0000-0002-6596-2601

\section{REFERENCES}

1. Kamekura N, Nitta Y, Takuma S, Fujisawa T. General anesthesia for a patient with pelizaeus-merzbacher disease. Anesth Prog 2016; 63: 91-4.

2. Numata Y, Gotoh L, Iwaki A, Kurosawa K, Takanashi J, Deguchi $\mathrm{K}$, et al. Epidemiological, clinical, and genetic landscapes of hypoymelinating leukodystrophies. J Neurol 2014; 261: 752-8.

3. Hobson GM, Garbern JY. Pelizaeus-merzbacher disease, pelizaeus-merzbacher-like disease 1 , and related hypomyelinating disorders. Semin Neurol 2012; 32: 62-7.

4. Hernández-Palazón J. Anaesthetic management in children with metachromatic leukodystrophy. Paediatr Anaesth 2003; 13: 7334.

5. Torii T, Miyamoto Y, Yamauchi J, Tanoue A. Pelizaeus-merzbach- er disease: cellular pathogenesis and pharmacologic therapy. Pediatr Int 2014; 56: 659-66.

6. Garbern JY. Pelizaeus-merzbacher disease: genetic and cellular pathogenesis. Cell Mol Life Sci 2007; 64: 50-65.

7. American Society of Anesthesiologists Committee. Practice guidelines for preoperative fasting and the use of pharmacologic agents to reduce the risk of pulmonary aspiration: application to healthy patients undergoing elective procedures: an updated report by the American Society of Anesthesiologists task force on preoperative fasting and the use of pharmacologic agents to reduce the risk of pulmonary aspiration. Anesthesiology 2017; 126: 376-93.

8. Tobias JD. Anaesthetic considerations for the child with leukodystrophy. Can J Anaesth 1992; 39: 394-7.

9. Mariotti P, Fasano A, Contarino MF, Della Marca G, Piastra M, Genovese O, et al. Management of status dystonicus: our experience and review of the literature. Mov Disord 2007; 22: 963-8.

10. Wang PJ, Hwu WL, Shen YZ. Epileptic seizures and electroencephalographic evolution in genetic leukodystrophies. J Clin Neurophysiol 2001; 18: 25-32.

11. Cooperman LH, Strobel GE Jr, Kennell EM. Massive hyperkalemia after administration of succinylcholine. Anesthesiology 1970; 32: 161-4.

12. Renier WO, Gabreëls FJ, Hustinx TW, Jaspar HH, Geelen JA, Van Haelst UJ, et al. Connatal pelizaeus-merzbacher disease with congenital stridor in two maternal cousins. Acta Neuropathol 1981; 54: 11-7.

13. Saugier-Veber P, Munnich A, Bonneau D, Rozet JM, Le Merrer M, Gil R, et al. X-linked spastic paraplegia and pelizaeus-merzbacher disease are allelic disorders at the proteolipid protein locus. Nat Genet 1994; 6: 257-62.

14. Arroyo EJ, Xu T, Grinspan J, Lambert S, Levinson SR, Brophy PJ, et al. Genetic dysmyelination alters the molecular architecture of the nodal region. J Neurosci 2002; 22: 1726-37.

15. Yamamoto T, Shimojima K. Pelizaeus-merzbacher disease as a chromosomal disorder. Congenit Anom (Kyoto) 2013; 53: 3-8. 